\title{
PREVALENCE AND SIDE EFFECTS OF CAFFEINE CONSUMPTION AMONGST MEDICAL STUDENTS.
}

\section{Kabir Bansal*}

Third year, MBBS, Punjab Institute of Medical Sciences, Jalandhar. ${ }^{*}$ Corresponding Author

House physician, Internal medicine, Government Medical College and Hospital, Patiala.

ABSTRACT Caffeine is the most widely consumed central nervous system stimulant in the world. Medical students and health professionals are more prone to consumption of high amounts of caffeinated products because they are regularly exposed to stress conditions. The current study is cross sectional study. The participants were selected on the basis of inclusion and exclusion criteria. Around 500 participants composed of the sample size who were distributed with the questionnaire and the responses were recorded. The study showed consumption of caffeinated products by major portion of the sample size. Major reasons of consumption being to feel more awake, mood elevation and focus and concentration. Various side effects faced were difficulty in sleep, frequent urination, tremors and palpitations. Long term consumption showed various withdrawal symptoms is many individuals like headache, fatigue and cravings. Awareness among medical students and health professionals regarding this topic is important as future healthcare lies in their hand.

\section{KEYWORDS : Caffeine, Coffee, Anxiety, Tea, Soft drink, Tremors, Restlessness.}

\section{INTRODUCTION}

Caffeine or 1,3,7-Trimethylpurine-2,6-dione is an alkaloid, naturally occurring in coffee beans, tea leaves, cola nuts, Yerba-mate leaves, cocoa beans and guarana seeds. Caffeine is the most widely consumed central nervous system stimulant in the world [1] and one of the most extensively studied elements in the human diet $[1,2]$. Caffeine can be ingested in foods made from natural sources such as coffee, tea, and chocolates [3]. However, synthetic caffeine is often added to food products and beverages to enhance their stimulant properties [3] Coffee has appeared to be the major source of caffeine, followed by soft drinks [3].

Different populations use caffeine for various reasons; for example, doctors and surgeons use caffeine to reduce fatigue and increase alertness [4], athletes use caffeine to enhance their physical performance [5], while young adults use caffeine to get more energy, or for the taste, or as part of social gathering or for image enhancement [6].

Medical student's life journey revolves around regular tests, internal exams and excessive stress, majorly due to sleepless working hours. Medical students have to put forth extra effort beyond their mental threshold and physical stress to show progress in their exams and career. The consumption of caffeinated products is one of the coping methods used by medical students as its habitual consumption has been significantly related to increased self-reported alertness, improved performance of vigilance tasks and fewer lapses of attention, improved long-term memory and faster locomotor speed [7][8] But this repetitive and prolonged used of high amount of caffeinated products in various forms leads to addiction and intoxication which can further have withdrawal effects also.

Approximately $90 \%$ of the caffeine contained in a cup of coffee is cleared from the stomach within 20 minutes after oral ingestion, with its effects commencing within an hour and lasting for three to four hours. [9] The peak plasma concentration is reached after approximately 40-60 minutes, [10] with a half-life of approximately six hours in healthy adults. [11] The half-life is shorter in smokers and longer in pregnant women and women taking oral contraceptives. [9]

Several studies showed that consuming a moderate amount of caffeine has a protective effect against cancer [12], diabetes mellitus type 2 [13], inflammatory diseases and pain [14], Parkinson's and related neurodegenerative diseases
[15], cardiovascular disease [16], and stroke [17]. Light to moderate caffeine intake has also been found to be associated with reduced risk of death [18] from all causes, including suicide [19]. However, intake of caffeine in high doses may lead to adverse effects on health [20]. A recent systematic review identified several unwanted symptoms associated with a high daily intake of caffeine; these include palpitations, headache, tremors, anxiety, agitation, restlessness, and sleep problems [21].

Taking all of the above information collectively, it becomes clear that more research is necessary to study the overall intake of caffeine and its potential cumulative effects on physiology and behaviour among populations (e.g. medical students) vulnerable to its negative effects.

\section{METHODOLOGY}

The cross-sectional research design was used to assess the prevalence of intake of caffeinated products and the side effects experienced by the consumers. The sample selection was based on Inclusion and Exclusion criterias.

Inclusion Criteria:

MBBS students (First prof-Interns), residents.

\section{Exclusion Criteria:}

Dental students, nursing staff, physicians, technicians.

The study was conducted by distribution of the questionnaire made via Google forms online. The link to the respective questionnaire was distributed to medical students through their respective college WhatsApp ${ }^{\circledR}$ groups. Data were collected by self-administered responses to the questionnaire which was developed after extensive literature review. Informed consent was taken from the participants before filling the questionnaire. Only the complete filled forms were selected and the incomplete forms were excluded automatically in data analysis.

The questionnaire consisted of structured, closed-ended questions. There were no open ended or continuing questions, making the questionnaire simple and quick to answer. Questions asked for information regarding demographic data (age, sex), amount and frequency of caffeinated products consumed, types of caffeinated products consumed, purpose or circumstances for consumption, various side effects and withdrawal effects experienced. The data was collected from the questionnaire and recorded manually. 
Data analysis was performed using IBM statistical package for social science (SPSS) software version 21.

\section{RESULTS}

A total of 500 medical students participated in the study. There were 185 (37\%) male candidates and 315 (63\%) female candidates. [Figurel.0] Students were mainly from age group of 20-22 year i.e. 217 candidates (43.4\%) followed by $23-25$ year group 135 candidates (27\%), 17-19 year group 118 candidates $(23.6 \%)$ and $26+$ year 30 candidates $(6 \%)$. [Figure 2.0] Out of total 500 candidates, $97 \%$ (485 students) accepted to consume caffeinated products in various forms and $3 \%$ (15 students) denied of any voluntary intake of caffeinated products.[Figure 3.0] Coffee was the most popular and frequently consumed caffeinated product. It's consumption was accepted by 385 students (77\%). Other products were Tea297 students (59\%), soft drink- 222 students (44\%), energy drink- 77 students (15\%) and others- 6 students (1\%). [Figure4.0] Other products with fractionated percentages consisted of caffeine pills, tablets and chewing-gums. 15 students $(3 \%)$ denied consumption of any type of caffeinated products. Regarding frequency of consumption of caffeinated products 204 students $(40.80 \%)$ did 2-3 times/24 hours, 123 students $(24.60 \%)$ did $1-2$ times/24 hours, 92 students (18\%) did 4-5 times/24 hours, 64 students(12.80\%) did 0-1 times/ 24 hours and only 2 students $(0.40 \%)$ did $\geq 6$ times $/ 24$ hours.[Figure 5.0] Although purpose of consumption of caffeinated products were enormous but because of similarities they were narrowed to the following main responses: to feel more awake $(38.40 \%)$, focus and concentration (25\%), mood elevation (18\%), to be more alert (15\%), improve physical performance (11.60\%) and others (5.20\%).[Figure6.0] However, the response of- no specific purpose remained the most answered i.e. by $43.40 \%$ candidates. Consumption of caffeinated products accompanied various side effects experienced by some fraction of the total sample. They were: insomnia (28\%), frequent urination $(18.80 \%)$, palpitations $(16.80 \%)$, tremors $(15.80 \%)$, stomach pain $(12 \%)$, restlessness $(5 \%)$ and others (3.40\%). [Figure7.0] However most of the candidates said that they never experienced any side effects of caffeinated products i.e. 244 students (48.80\%). At last candidates were asked if they experienced any withdrawal effects if they didn't get they daily dose of caffeine and many of them did responded positive with the withdrawal symptoms. They were: fatigue $(13 \%)$, drowsiness $(10.40 \%)$, anxiety $(5.60 \%)$, sick stomach $(0.80 \%)$, headache $(18.00 \%)$, cravings $(13 \%)$, irritability (3.80\%) and others (1.80\%). [Figure 8.0] However almost half of the candidates answered that they never experienced any kind of withdrawal effects i.e. 215 students (43\%).

\section{DISCUSSION}

Age old tradition and our assumptions has made consumption of caffeinated products as a part of our meal system, whether the consumption assists the nutritional benefit or worsen the life state of individual is yet to be scrutinized. The general lack of research relating to the role of caffeinated products on the day to day activities among the medical students is an area that the current paper will try to discuss.

The present study showed that $97 \%$ of the participants admitted about the consumption of caffeinated products in varies forms, out of which coffee was the most popular one, being consumed by $77 \%$ of the participants. Other popular choices were tea, soft drink and energy drinks. Some unpopular products where caffeine tablets, chewing gums etc. As the study sample included medical students and residents therefore most of the participants belonged to early 20 s of age. In the present study it was found that $84 \%$ of students consumed caffeinated products daily as their normal routine. However, as many as $60 \%$ of the participants consumed caffeinated products multiple times in a single day. The main purposes of consumption of were to feel more awake (38.40\%), increase focus and concentration (25\%) and mood elevation (18\%). Although $48.8 \%$ of candidates denied for any reaction or side effects after consumption of caffeinated products but other revealed some of them. Some of the most commonly experienced were insomnia like features (28\%), frequent urination (18\%), palpitations $(16.80 \%)$, fine tremors $(15.80 \%)$ and stomach pain (12\%). A good proportion of the participants who have been consuming these products multiple times in a single day complained of various withdrawal symptoms if they didn't get their daily dose of the caffeine. Some of the commonly experienced symptoms were headache (18\%), cravings for the product (13\%), fatigue (13\%) and drowsiness $(10.40 \%)$. However, $43 \%$ of the candidates denied of any major withdrawal symptoms even if they were abstained from caffeinated products.

\section{CONCLUSION}

The present study described the pattern of consumption of caffeinated products among the medical students and the related effects on the body experienced by them. Medical students and Health professionals are faced with regular high stress. To get rid of the same, they try various things to cope up, out of which consumption of caffeinated products is very popular and common. They need to be aware of the consequences of consumption of these products. Although most of the products are marketed as safe to consume, but regular and high amount of consumption can lead to various effects on the human body. The future healthcare relies on today's medical students, who are in a responsible position to provide complete education to the community about the consequences of consuming caffeinated products. Instead of these products various alternative healthy options should be considered for stress management. Medical students should be sensitized with stress management workshops and awareness programs about alternative nutrients filled diet for their long-lasting health benefits.

\section{DECLARATION OF CONFLICTING INTERESTS: NONE}

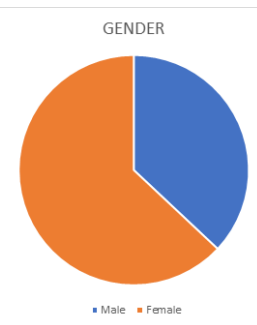

Figure 1.0

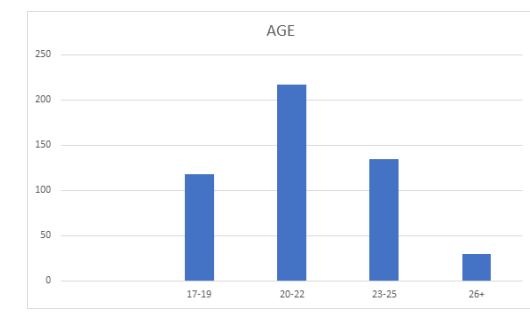

Figure 2.0

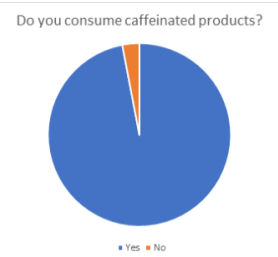

Figure 3.0 
In what form do you consume caffeinated products?

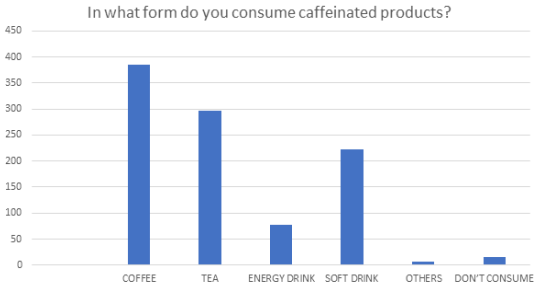

Figure 4.0

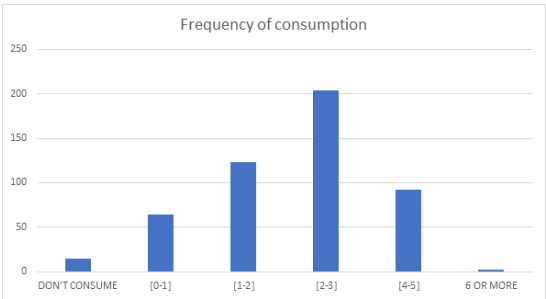

Figure 5.0

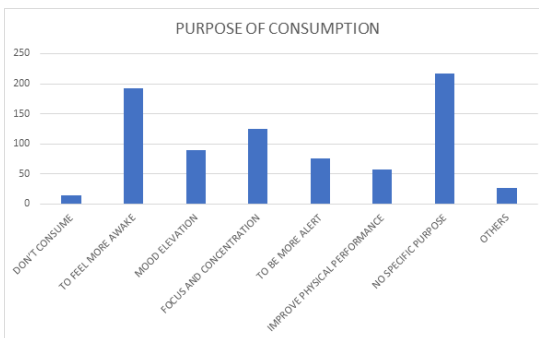

Figure 6.0

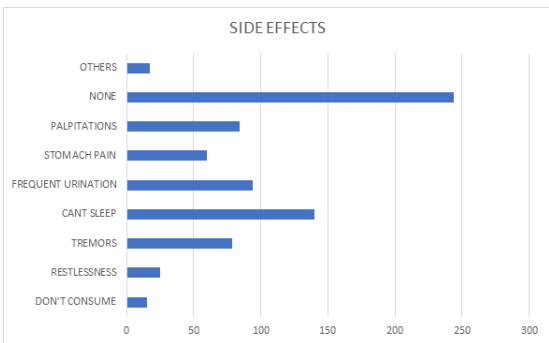

Figure 7.0

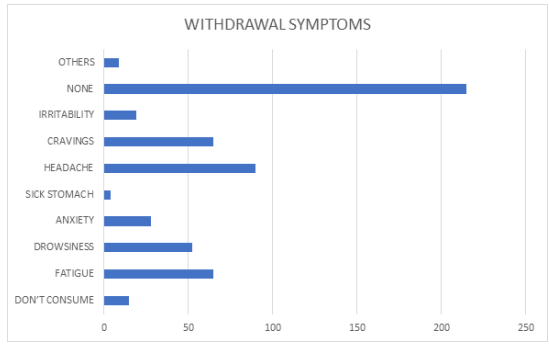

\section{Figure 8.0}

\section{REFERENCES}

1. Martyn, D.; Lau, A.; Richardson, P; Roberts, A. Temporal patterns of caffeine intake in the United States. Food Chem. Toxicol. 2018, 111,71-83.

2. Ahluwalia, N.; Herrick, K. Caffeine Intake from Food and Beverage Sources and Trends among Children and Adolescents in the United States: Review of National Quantitative Studies from 1999 to 201112345 . Adv. Nutr. 2015, 6, 102-111.

3. Mitchell, D.C.; Knight, C.A.; Hockenberry, J.; Teplansky, R.; Hartman, T.J. Beverage caffeine intakes in the US. Food Chem. Toxicol. 2014, 63, 136-142

4. Franke, A.G.; Bagusat, C.; McFarlane, C.; Tassone-Steiger, T.; Kneist, W.; Lieb, K. The use of caffeinated substances by surgeons for cognitive enhancement. Ann. Surg. 2015, 261, 1091-1095

5. Desbrow, B.: Leveritt, M.Well-trained endurance athletes' knowledge, insight, and experience of caffeine use. Int. J. Sport Nutr. Exerc. Metab. 2007, 17, 328-339

6. Turton, P.; Piché, L.; Battram, D.S. Adolescent attitudes and beliefs regarding caffeine and the consumption of caffeinated beverages. J. Nutr. Educ. Behav. $2016,48,181-189$ el.
7. Christopher G, Sutherland D, Smith A. Effects of caffeine in non-withdrawn volunteers. Human Psychopharmacology 2005;20:47-53.

8. Hameleers PA, Van Boxtel MP, Hogervorst E, et al. Habitual caffeine consumption and its relation to memory, attention, planning capacity and psychomotor performance across multiple age groups. Human Psychopharmacology 2000;15:573-81.

9. Winston AP, Hardwick E, Jaberi N. Neuropsychiatric effects of caffeine. Advances in Psychiatric Treatment 2005; 11:432-9.

10. James JE. Critical review of dietary caffeine and blood pressure: a relationship that should be taken more seriously. Psychosom Med 2004;66:63-71.

11. James JE, Rogers PJ. Effects of caffeine on performance and mood: withdrawal reversal is the most plausible explanation. Psychopharmacology 2005; 182:1-8.

12. Arab, L. Epidemiologic evidence on coffee and cancer. Nutr. Cancer 2010,62, 271-283.

13. Muley, A.; Muley, P.; Shah, M. Coffee to reduce risk of type 2 diabetes? A systematic review. Curr. Diabetes Rev. 2012, 8, 162-168.

14. Paiva, C.; Beserra, B.; Reis, C.; Dorea, J.G.; Da Costa, T.; Amato, A.A. Consumption of coffee or caffeine and serum concentration of inflammatory markers: A systematic review. Crit. Rev. Food Sci. Nutr. 2019, 59, 652-663.

15. Ross,G.W.; Abbott, R.D.; Petrovitch, H.; Morens, D.M.; Grandinetti, A.; Tung K.H.; Tanner, C.M.; Masaki, K.H.; Blanchette, P.L.; Curb, J.D.; et al. Association of coffee and caffeine intake with the risk of Parkinson disease. JAMA 2000 $283,2674-2679$

16. Bidel, S.; Tuomilehto, J. The Emerging Health Benefits of Coffee with an Emphasis on Type 2 Diabetes and Cardiovascular Disease. Eur. Endocrinol. 2013, 9, 99-106.

17. Kim, B.; Nam, Y.; Kim, J.; Choi, H.; Won, C. Coffee Consumption and Stroke Risk: A Meta-analysis of Epidemiologic Studies. Korean J. Fam. Med. 2012, 33, 356-365.

18. Zhao, Y.;Wu, K.; Zheng, J.; Zuo, R.; Li, D. Association of coffee drinking with all-cause mortality: A systematic review and meta-analysis. Public Health Nutr. 2015, 18, 1282-1291.

19. Lucas, M.; O'Reilly, E.J.; Pan, A.; Mirzaei, F.Willett,W.C.; Okereke, O.I. Ascherio, A. Coffee, caffeine, and risk of completed suicide: Results from 3 prospective cohorts of American adults. World J. Biol. Psychiatry 2014, 15, 377-386.

20. Bühler, E.; Lachenmeier, D.W.; Schlegel, K.;Winkler, G. Development of a tool to assess the caffeine intake among teenagers and young adults. Ernahr. Umsch. 2014, 61, 58-63.

21. Temple, J.L.; Bernard, C.; Lipshultz, S.E.; Czachor, J.D.; Westphal, J.A.; Mestre, M.A. The Safety of Ingested Caffeine: A Comprehensive Review. Front. Psychiatry 2017, 8. 